\title{
Effect of torsion on the undrained limiting lateral resistance of piles
} in clay

Konstantinos Georgiadis ${ }^{1}$ and Brian Sheil ${ }^{2}$

Initial submission, 29 January 2019

Revision \#1, 26 April 2019

Revision \#2,

Main text word count: 5697

Tables: 4

Figures: 11

\footnotetext{
${ }^{1}$ Dipl(Eng), MSc, DIC, PhD. Department of Civil Engineering, Aristotle Univ. of Thessaloniki, 541 24, Thessaloniki, Greece. kgeorg@civil.auth.gr.

${ }^{2}$ BE, PhD. Department of Engineering Science, University of Oxford, Parks Road, Oxford, OX1 3PJ, UK. brian.sheil@eng.ox.ac.uk.
} 


\section{Abstract}

2 The behaviour of piles subjected to lateral loads has been explored by a number of

3 investigators over the past five decades. However, the vast majority of previous

4 literature have ignored the potential influence of additional torsional effects due to

5 horizontal eccentricities of the applied lateral loads. In this paper, an upper bound mechanism and a lower bound stress field are presented for the calculation of the

7 ultimate combined torsional and lateral loads on single piles in clay. The

8 corresponding analytical upper and lower bound plasticity solutions are shown to be

9 in excellent agreement, bracketing the theoretically exact failure loads with an error of

10 less than $0.5 \%$. Two-dimensional finite element analyses are also presented and the

11 computed ultimate loads and observed failure mechanisms are shown to compare very

12 well with the plasticity solutions. Based on the numerical results and analytical

13 solutions, failure load interaction diagrams and a simple empirical equation are

14 presented that allow the calculation of the lateral bearing capacity factor for any level

15 of applied torsion and pile-soil adhesion factor. It is demonstrated that the effect of

16 torsion can be very significant, causing reduction in lateral bearing capacity of up to

$1750 \%$ and therefore should be considered in design.

18

19 Keywords: Piles \& piling, Bearing capacity, Torsion, Theoretical analysis, Plasticity, 20 Finite-element modelling 


\section{Introduction}

Pile foundations are one of the most widely-adopted onshore and offshore foundation system. For structures subjected to environmental loading such as wind and waves, this commonly results in considerable lateral loads being transferred to the supporting foundations (Byrne and Houlsby, 2003). Significant displacements are often required to mobilise the ultimate lateral capacity and for this reason the focus of pile design has shifted towards serviceability limit state (Reddy and Stuedlein, 2017). However, rigorous serviceability limit state design, considering soil stiffness nonlinearity, is predicated on an accurate estimation of ultimate capacity (Sheil et al., 2018). A robust assessment of pile capacity therefore remains a key issue in the design of onshore and offshore pile foundation systems (Randolph, 2003).

The lateral capacity of a single pile in undrained soil has been the subject of a number of analytical (e.g. Murff \& Hamilton, 1993; Yu et al., 2015), numerical (e.g. Brown \& Shie, 1991; Yang \& Jeremic, 2002; Georgiadis \& Georgiadis, 2010; Zhang \& Andersen, 2019), and experimental (e.g. Matlock, 1970; Brown et al. 1988; Jeanjean, 2009; Zhang et al., 2010; Nimityongskul et al., 2017) investigations. Using the upper bound theory of plasticity, Murff and Hamilton (1993) noted that although the ultimate lateral resistance of a pile in undrained soil increased with depth, it eventually reached a limiting value at a 'critical depth'. Below the critical depth, the limiting pressure corresponds to a plane strain 'flow-around' failure mechanism and depends exclusively on the undrained shear strength of the soil and the pile-soil adhesion. Solutions for this flow-around limiting pressure have been proposed by Randolph and Houlsby (1984), Murff et al. (1989) and Martin and Randolph (2006). 
By contrast, the potential influence of additional torsional effects, due to horizontal eccentricities of the applied lateral loads, on lateral pile capacity has largely been ignored. Herrera (2001), Hu (2003) and Hu et al. (2006) reported on the results of centrifuge tests of piles in sand subjected to various applied torsional-lateral loads. Thiyyakkandi et al. $(2016,2017)$ reported the results of a full-scale field testing programme to assess the behaviour of drilled shafts and jetted and grouted piles, respectively, to lateral-torsional loads. In all cases, these investigators reported that the lateral resistance is significantly reduced (up to 87\%) by simultaneous application of torsion. It seems prudent, therefore, that the potential development of torsional loads, and their effect on lateral pile capacity, should be considered in design.

This paper explores the effect of torsion on the undrained limiting lateral resistance of a single pile. Plane strain conditions associated with deep flow-around failure are considered. The ultimate lateral resistance is determined using finite element analysis (FEA) for different levels of applied torsion and adhesion factor. An upper bound mechanism and a lower bound stress field are subsequently presented. Based on the numerical results and analytical solutions, failure load interaction diagrams and a simple empirical equation are presented that allow the calculation of the lateral bearing capacity factor for any level of applied torsion and pile-soil adhesion factor.

\section{Problem definition}

A description of the problem considered in this paper is presented in Figure 1. A pile with diameter $D$ is considered embedded in undrained soil. The pile is either simultaneously translated with velocity $v_{0}$ and rotated with angular velocity $\omega_{0}$, or subjected to combined lateral load $P$ and torque $T$ per unit length of the pile, 
depending on the type of analysis performed (displacement finite element analysis, upper bound limit analysis or lower bound limit analysis). The soil is assumed rigid perfectly plastic in the upper and lower bound analyses and elastic - perfectly plastic in the finite element analyses. Adhesion factors $\alpha$ (ratio of the interface adhesion $\tau_{\mathrm{i}}$ to the undrained strength of the soil mass $s_{\mathrm{u}}$ ) ranging from 0 (smooth) to 1 (rough) are considered for the pile - soil interface. It is worth remarking that in the extreme case of a smooth interface $(\alpha=0)$, the pile fails by pure rotation for an infinitesimally small value of applied torque $T$, although it still has a lateral capacity.

Table 1: Parameter normalisation adopted in this study

\begin{tabular}{|l|c|}
\hline Variable & $\begin{array}{c}\text { Dimensionless / } \\
\text { normalised form }\end{array}$ \\
\hline Lateral bearing capacity factor, $N_{\mathrm{p}}$ & $\frac{P}{S_{\mathrm{u}} D}$ \\
\hline Torsional bearing capacity factor, $N_{\mathrm{T}}$ & $\frac{T}{S_{\mathrm{u}} D^{2}}$ \\
\hline Normalised lateral load, $n_{\mathrm{p}}$ & $\frac{P}{P_{\mathrm{u}}}=\frac{N_{\mathrm{p}}}{N_{\mathrm{pu}}}$ \\
\hline Normalised torque, $n_{\mathrm{T}}$ & $\frac{T}{T_{\mathrm{u}}}=\frac{N_{\mathrm{T}}}{N_{\mathrm{Tu}}}$ \\
\hline
\end{tabular}

9

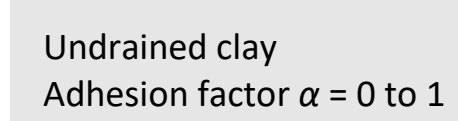


81 Figure 1: Schematic plan view of a pile section embedded in undrained clay Definition of failure loads and associated translational and rotational velocities. All results and discussions in this paper are presented in terms of dimensionless $\left(N_{\mathrm{p}}\right.$, $\left.N_{\mathrm{T}}\right)$ and normalised $\left(n_{\mathrm{p}}, n_{\mathrm{T}}\right)$ loads defined in Table 1 . The normalisations use the ultimate lateral load per unit length and bearing capacity factor for purely-lateral loading ( $P_{\mathrm{u}}$ and $N_{\mathrm{pu}}$ respectively) and the ultimate torsional load per unit length and bearing capacity factor for purely-torsional loading ( $T_{\mathrm{u}}$ and $N_{\mathrm{Tu}}$ respectively).

\section{Finite element analyses}

\subsection{Finite element mesh and material parameters}

A series of plane strain FEA were performed with the finite element program PLAXIS 2D (Brinkgreve et al. 2018) in order to determine the ultimate loads and the associated failure mechanisms. The finite element mesh is shown in Figure 2a. It consists of 12876 15-noded triangular elements and has dimensions $10 \mathrm{~m} \times 10 \mathrm{~m}$. All boundaries of the computational domain were restricted from movement normal to the respective surface. A detail of the finite element mesh around the pile is shown in Figure $2 \mathrm{~b}$. A total of 112 15-noded triangular elements are in contact with the pile (520 nodes along the pile perimeter). A $1 \mathrm{~m}$ diameter pile was considered in all cases. In order to apply moment loading and to ensure that the pile behaves rigidly, beam elements with axial stiffness $E A=15 \cdot 10^{6} \mathrm{kN} / \mathrm{m}$ and bending stiffness $E I=5 \cdot 10^{5}$ $\mathrm{kNm}^{2} / \mathrm{m}$ were placed along the pile perimeter and two mutually orthogonal diameters, as shown in Figure 2a. The soil was modelled as a weightless linear elastic - perfectly plastic material with Tresca shear strength criterion and typical undrained soil parameters: undrained Young's modulus $E_{\mathrm{u}}=20 \mathrm{MPa}$, undrained Poisson's ratio $v_{\mathrm{u}}=$ 0.495 and undrained shear strength $s_{\mathrm{u}}=50 \mathrm{kPa}$. It is worth noting that the 
dimensionless and normalized loads presented in the following sections are

107 independent of the selection of the above parameters. Interface elements were placed

108 between the pile and the soil. The interface was also modelled as an elastic perfectly-

109 plastic Tresca material with the same properties of the soil except a strength of $\tau_{\mathrm{i}}=$ $\alpha \cdot s_{\mathrm{u}}$ was adopted.
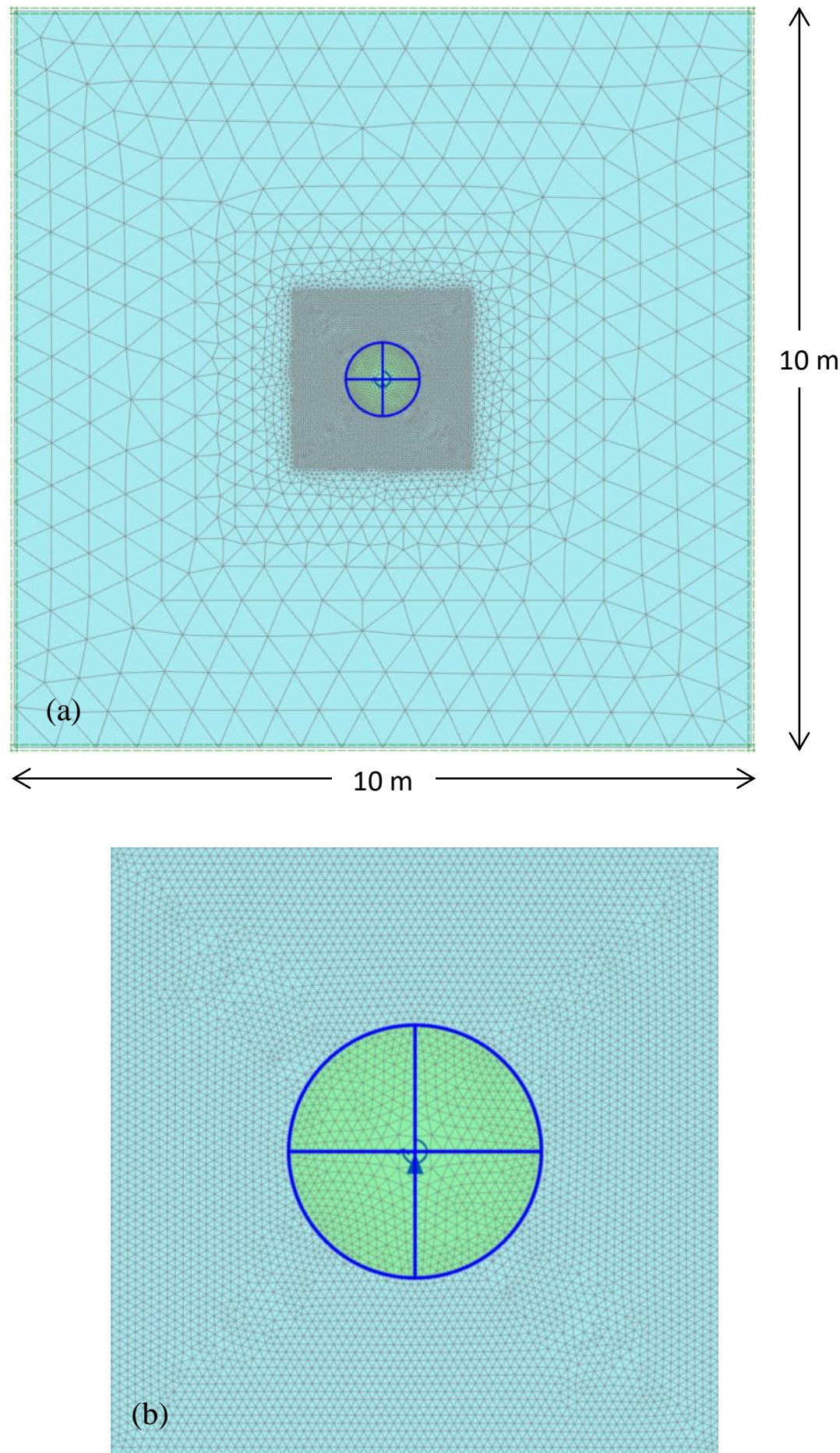
Figure 2: (a) Finite element mesh adopted for this study showing increased mesh density in zone immediately surrounding pile; 12876 elements. (b) Detail of mesh around the pile.

\subsection{Finite element calculation stages}

The calculation stages adopted in the finite element analyses are as follows:

1. 'Wished in place' installation of a weightless pile.

2. Application of a purely-torsional load, a purely-lateral load or a combination thereof until pile failure was achieved. The directions of the applied loads are shown in Figure 1.

\subsection{Validation}

The parameters $N_{\mathrm{pu}}$ and $N_{\mathrm{Tu}}$ were first determined by loading the pile, either laterally or torsionally, to failure for different values of $\alpha$. The ultimate bearing capacity factors predicted using FEA are summarized in Table 2 and are compared with those determined analytically using:

(a) The lower bound equation developed by Randolph and Houlsby (1984) for laterally loaded piles, which has subsequently been shown to be practically exact (Martin and Randolph, 2006):

$$
\begin{aligned}
N_{\mathrm{pu}}=\pi+2 \arcsin (\alpha)+2 \cos (\arcsin (\alpha)) \\
+4\left[\cos \left(\frac{\arcsin (\alpha)}{2}\right)+\sin \left(\frac{\arcsin (\alpha)}{2}\right)\right]
\end{aligned}
$$

(b) A simple limit equilibrium calculation for purely-torsional loading:

$$
N_{\mathrm{Tu}}=\alpha \frac{\pi}{2}
$$

It can be seen that very good agreement exists between the present FEA results and the analytical solutions. 
Table 2: Ultimate bearing capacity factors for different values of $\alpha$ for purely-lateral

\subsection{Combined lateral-torsional loading}

\begin{tabular}{|c|c|c|c|c|}
\hline \multirow{2}{*}{$\begin{array}{c}\text { Adhesion } \\
\text { factor } \alpha\end{array}$} & \multicolumn{2}{|c|}{$\begin{array}{c}\text { Ultimate lateral } \\
\text { bearing capacity } \\
\text { factor } N_{\text {pu }}\end{array}$} & \multicolumn{2}{|c|}{$\begin{array}{c}\text { Ultimate torsional } \\
\text { bearing capacity } \\
\text { factor } N_{\text {Tu }}\end{array}$} \\
\cline { 2 - 5 } & FEA & Eq. (1) & FEA & Eq. (2) \\
\hline 0 & 9.18 & 9.14 & 0 & 0 \\
\hline 0.25 & 10.12 & 10.06 & 0.393 & 0.393 \\
\hline 0.5 & 10.85 & 10.82 & 0.785 & 0.785 \\
\hline 0.75 & 11.48 & 11.45 & 1.178 & 1.178 \\
\hline 1 & 11.96 & 11.94 & 1.571 & 1.571 \\
\hline
\end{tabular}

To explore the influence of $n_{\mathrm{T}}$ on the lateral bearing capacity factor, $N_{\mathrm{p}}$, and

associated failure mechanisms, analyses were performed in which a torque was first applied to the pile followed by lateral loading to failure. The incremental

displacements at failure determined for different values of the adhesion factor $\alpha(0.25$, $0.5,0.75$ and 1$)$ and normalised torque $n_{\mathrm{T}}(0,0.5$ and 0.95$)$ are presented in Figure 3.

For $n_{\mathrm{T}}=1$ (pure rotation), failure is confined to the pile-soil interface and is therefore not shown in this and subsequent figures. This mode of failure is the same irrespective of the value of the adhesion factor and the magnitude of lateral load. The calculated values of $N_{\mathrm{p}}$ for each case are also superimposed on the plot.

Apart from the expected loss of four-fold symmetry of the failure mechanism, the application of torque also reduces the size of the failure surface, as shown in Figure 3. The reduction in the size of the failure surface is dependent on the adopted value of $\alpha$ : a maximum reduction occurs for $\alpha=1$ and is less for smaller values of $\alpha$. As the

159 lateral capacity is governed by the size of the failure mechanism, similar observations can be made for the influence of $n_{\mathrm{T}}$ on $N_{\mathrm{p}}$. For example, an increase in $n_{\mathrm{T}}$ from 0 to 

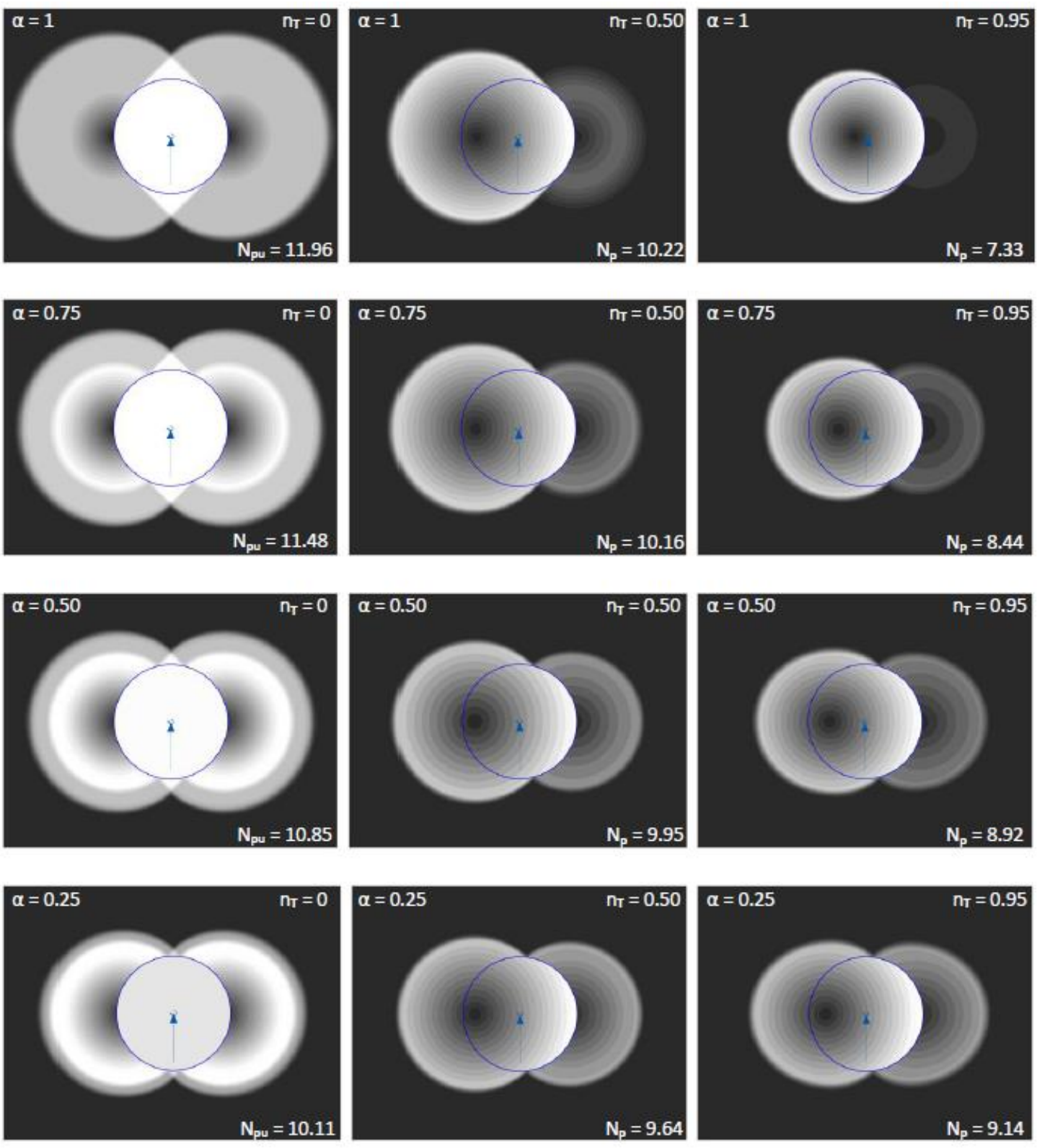

Figure 3: Contours of incremental soil displacements at failure for a laterally loaded pile with different levels of applied torsion; $\alpha=0.25,0.5,0.75$ and 1 . 

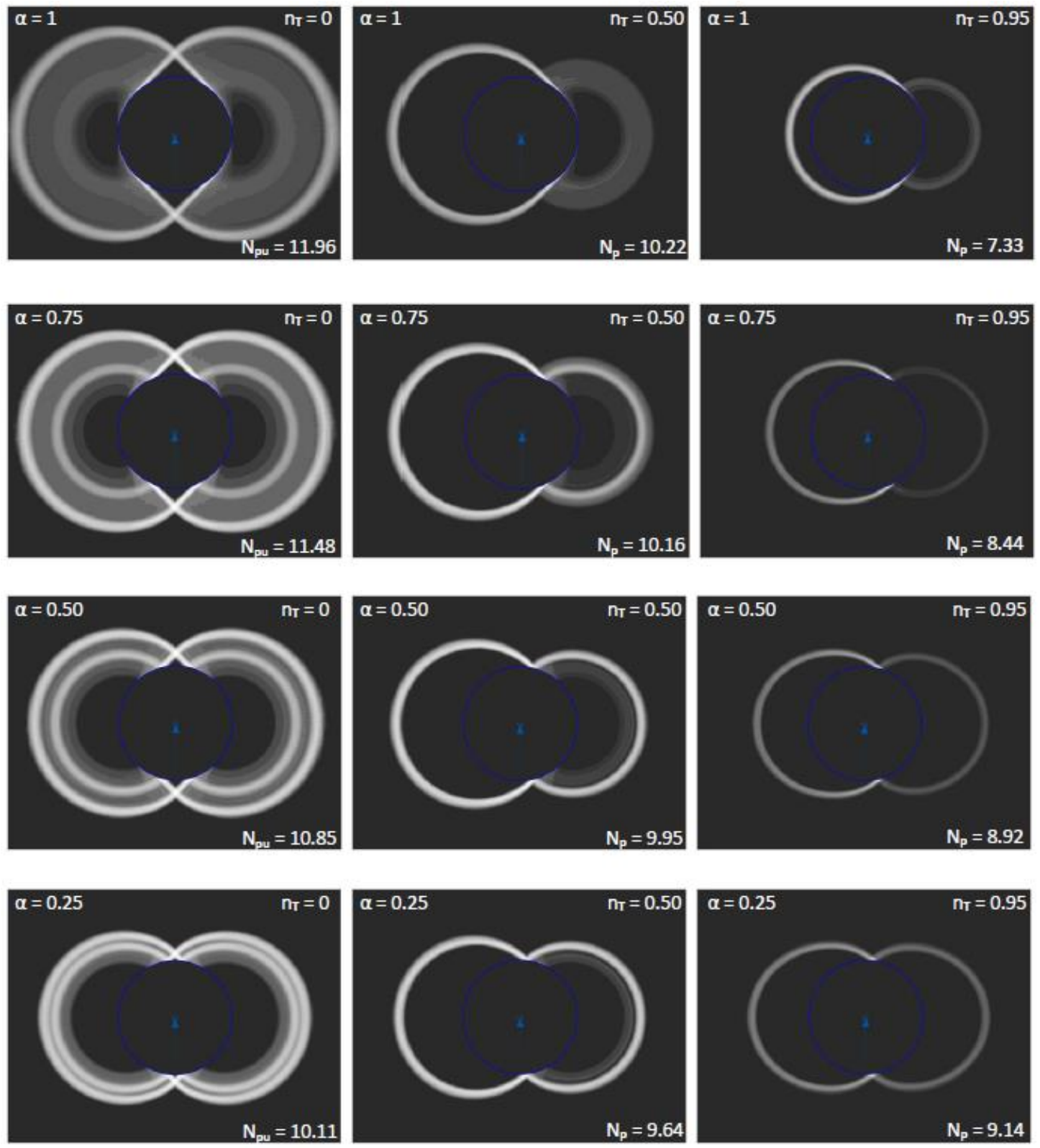

Figure 4: Contours of incremental shear strains at failure for a laterally loaded pile with different levels of applied torsion; $\alpha=0.25,0.5,0.75$ and 1 .

173 The incremental shear strains at failure plotted in Figure 4 show that no plastic

174 deformation occurs within this circular region of soil. Furthermore, as seen in Figure

1753 , no slip occurs along the section of the pile-soil interface that separates this region

176 from the pile. It therefore follows that this soil region and the pile behave as a single

177 rotating rigid body. The right-hand part of the mechanism involves both rigid body 
rotation and shear deformation of the soil and is similar to the mechanism observed for purely lateral loading $\left(n_{\mathrm{T}}=0\right)$ with one exception: there is no formation of a rigid triangular wedge at the front and back of the pile. For values of $n_{\mathrm{T}}$ close to 1 combined with small values of $\alpha$, the right-hand side of the mechanism devolves into a purely rotational mechanism similar to the left-hand side.

\section{Upper bound failure mechanism}

Based on the failure mechanisms identified using FEA (discussed above), the mechanism shown in Figure 5 (denoted 'Mechanism I') is used to determine the upper bound of the ultimate lateral load, $P$, and torsional load, $T$. The mechanism is geometrically symmetrical about the horizontal axis $\mathrm{x}$. The horizontal axis is also an axis of anti-symmetry with respect to velocities. On the left-hand side, the mechanism consists of a rigid body $\mathrm{ABCHC}$ 'B' $\mathrm{A}$ which includes the whole pile and the soil inside the circular velocity discontinuity $\mathrm{CHC}^{\prime}$ of radius $r_{x}$ (see Figure 5). No slip takes place at the pile-soil interface segment CGC'. This rigid body rotates about point $\mathrm{X}$ with angular velocity $\omega_{0}$, and causes the displacement and deformation of the soil on the right-hand side of the pile, as shown in Figure 5.

The right-hand side of the mechanism is based on the mechanism proposed by Martin and Randolph (2006) for a laterally loaded single pile and is similar to the mechanisms used by Georgiadis et al. (2013a,b) for laterally loaded two-pile groups and pile rows. It comprises a rigid region $\mathrm{ABFB}$ 'A that rotates about point $\mathrm{O}$ and a plastically deforming region CDED'C'B'FB. While the geometry of the right-hand side of the proposed mechanism is similar to previous solutions, the velocities are different because the mode of pile movement is combined translational and rotational 


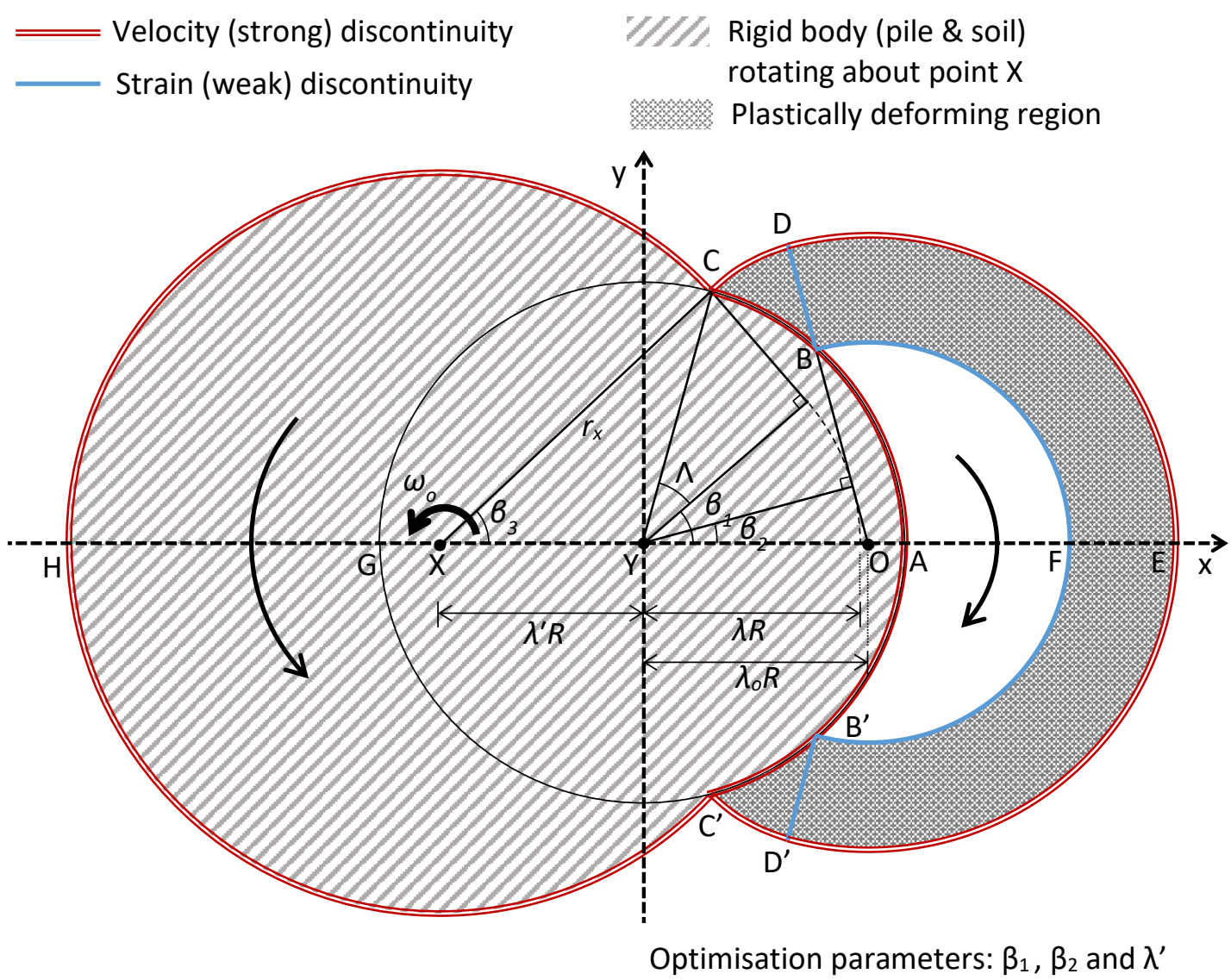

Figure 5: Upper bound failure mechanism for a pile subjected to combined lateraltorsional loading

rather than purely translational. This consequently affects the associated work calculations.

207 Three optimisation parameters define the geometry of the present mechanism: angles

$208 \beta_{1}$ and $\beta_{2}$ define the extent of the right-hand part of the mechanism and the

209 intersection points ( $\mathrm{C}$ and $\left.\mathrm{C}^{\prime}\right)$ of the two mechanisms; parameter $\lambda^{\prime}$ 'controls the position $(\mathrm{X})$ of the centre of the circular velocity discontinuity $\mathrm{CHC}^{\prime}$ and therefore the size of the rotating rigid region $\mathrm{ABCHC}$ 'B' $\mathrm{A}$. Angles $\beta_{3}$ and $\Lambda$, normalised distances

$212 \lambda$ and $\lambda_{\mathrm{o}}$, and the radius of the left-hand part of the mechanism $r_{\mathrm{x}}$ (defined in Figure

213 5), can be expressed as functions of the three optimisation parameters: 


$$
\begin{aligned}
& \Lambda=\arccos (\lambda) \\
& \lambda=\cos \left(\frac{\arccos (\alpha)}{2}\right) \\
& \beta_{3}=\arctan \left(\frac{\sin \left(\beta_{1}+\Lambda\right)}{\lambda^{\prime}+\cos \left(\beta_{1}+\Lambda\right)}\right) \\
& \lambda_{\mathrm{o}}=\frac{\lambda}{\cos \left(\beta_{2}\right)} \\
& r_{\mathrm{x}}=R \frac{\sin \left(\beta_{1}+\Lambda\right)}{\sin \left(\beta_{3}\right)}
\end{aligned}
$$

214 The lengths of the associated velocity discontinuities can also be expressed as

215 functions of the optimisation parameters and are presented in Table 3. The ultimate

216 lateral load $P$ is determined for a given $T / P$ ratio by equating the work done by the

217 external forces $\Delta W_{\mathrm{f}}$ to the work done by the internal stresses $\Delta W_{\mathrm{p}}$. For an angular

218 velocity $\omega_{\mathrm{o}}$ of the mechanism at point $\mathrm{X}$, the centre of the pile $\mathrm{Y}$ rotates with angular

219 velocity $\omega_{0}$ and translates with velocity $v_{0}$ defined as follows:

$$
\mathrm{v}_{\mathrm{o}}=\omega_{\mathrm{o}} \lambda^{\prime} R
$$

Consequently, the work done by the external forces $\Delta W_{\mathrm{f}}$ can be written as:

$$
\Delta W_{\mathrm{f}}=\mathrm{v}_{\mathrm{o}} P\left(1+\frac{T}{P} \cdot \frac{1}{\lambda^{\prime} R}\right)
$$

221 The work done by the internal stresses is equal to the sum of (a) the work done along each velocity discontinuity, which is a function of the velocity jump at the discontinuity, and (b) the work done within the plastically deforming regions CDB, C'D'B' and DED'B'FB, which is a function of the velocities of each region. The 225 velocity jumps along discontinuities $\mathrm{AB}\left(\Delta \mathrm{v}_{\mathrm{AB}}\right)$ and $\mathrm{BC}\left(\Delta \mathrm{v}_{\mathrm{BC}}\right)$, and the velocities within regions $\mathrm{CDB}, \mathrm{C}^{\prime} \mathrm{D}$ 'B' and $\mathrm{DED}$ 'B'FB $\left(\mathrm{v}_{\mathrm{s}, \mathrm{BC}}\right)$ can be obtained from the geometry of Figures 6(a) and 6(b) and are presented in Table 3 together with the 
Table 3: Calculation of work done by internal stresses

\begin{tabular}{|c|c|c|c|}
\hline $\begin{array}{l}\text { Discontinuity/ } \\
\text { region }\end{array}$ & Length & $\begin{array}{l}\text { Velocity jump }(\Delta \mathrm{v}) \\
\text { or velocity }(\mathrm{v})\end{array}$ & Work \\
\hline $\mathrm{B}^{\prime} \mathrm{AB}$ & $2 R\left(\beta_{2}+\Lambda\right)$ & $\mathrm{v}_{\mathrm{o}}\left(\frac{1}{\lambda_{\mathrm{o}}}+\frac{1}{\lambda^{\prime}}\right)$ & $2 \alpha s_{u} R \mathrm{v}_{\mathrm{o}}\left(\frac{1}{\lambda_{\mathrm{o}}}+\frac{1}{\lambda^{\prime}}\right)\left(\beta_{2}+\Lambda\right)$ \\
\hline $\mathrm{BC}$ and $\mathrm{B}^{\prime} \mathrm{C}^{\prime}$ & $R\left(\beta_{1}-\beta_{2}\right)$ & $\mathrm{v}_{\mathrm{o}}\left(\frac{\cos \beta}{\lambda}+\frac{1}{\lambda^{\prime}}\right)$ & $2 \alpha s_{u} R v_{0}\left(\frac{\sin \beta_{1}-\sin \beta_{2}}{\lambda}+\frac{\beta_{1}-\beta_{2}}{\lambda^{\prime}}\right)$ \\
\hline CD and C'D' & $R\left(\beta_{1}-\beta_{2}\right)\left[\sin \Lambda+\frac{\lambda}{2}\left(\beta_{1}-\beta_{2}\right)\right]$ & $\mathrm{v}_{\mathrm{o}} \frac{\sin \left(\beta_{1}+\Lambda\right)}{\lambda}$ & $2 s_{u} R \mathrm{v}_{\mathrm{o}} \frac{\sin \left(\beta_{1}+\Lambda\right)}{\lambda}\left(\beta_{1}-\beta_{2}\right)\left[\sin \Lambda+\frac{\lambda}{2}\left(\beta_{1}-\beta_{2}\right)\right]$ \\
\hline DED' & $2 R\left(\frac{\pi}{2}+\beta_{2}\right)\left[\lambda\left(\beta_{1}-\beta_{2}+\tan \beta_{2}\right)+\sin \Lambda\right]$ & $\mathrm{v}_{\mathrm{o}} \frac{\sin \left(\beta_{1}+\Lambda\right)}{\lambda}$ & $2 s_{u} R \mathrm{v}_{\mathrm{o}} \frac{\sin \left(\beta_{1}+\Lambda\right)}{\lambda}\left(\frac{\pi}{2}+\beta_{2}\right)\left[\lambda\left(\beta_{1}-\beta_{2}+\tan \beta_{2}\right)+\sin \Lambda\right]$ \\
\hline $\mathrm{CHC}^{\prime}$ & $2 R\left(\pi-\beta_{3}\right) \frac{\sin \left(\beta_{1}+\Lambda\right)}{\sin \beta_{3}}$ & $\mathrm{v}_{\mathrm{o}} \frac{\sin \left(\beta_{1}+\Lambda\right)}{\lambda^{\prime} \sin \beta_{3}}$ & $2 s_{u} R v_{o}\left(\frac{\sin \left(\beta_{1}+\Lambda\right)}{\sin \beta_{3}}\right)^{2} \frac{\left(\pi-\beta_{3}\right)}{\lambda^{\prime}}$ \\
\hline 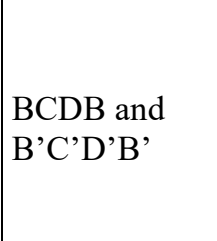 & - & $\mathrm{v}_{\mathrm{o}} \frac{\sin (\beta+\Lambda)}{\lambda}$ & $\begin{aligned} 2 s_{u} R \mathrm{v}_{\mathrm{o}}\left[\sin \left(\beta_{1}+\right.\right. & \Lambda)\left(2-\tan \Lambda \cdot\left(\beta_{1}-\beta_{2}\right)-\frac{\left(\beta_{1}-\beta_{2}\right)^{2}}{2}\right) \\
& -\cos \left(\beta_{1}+\Lambda\right)\left(\tan \Lambda+2\left(\beta_{1}-\beta_{2}\right)\right)-2 \sin \left(\beta_{2}+\Lambda\right) \\
& \left.+\tan \Lambda \cdot \cos \left(\beta_{2}+\Lambda\right)\right]\end{aligned}$ \\
\hline DED'B'FBD & - & $\mathrm{v}_{\mathrm{o}} \frac{\sin (\beta+\Lambda)}{\lambda}$ & $\begin{aligned} 2 s_{u} R v_{\mathrm{o}}\left(\frac{\pi}{2}+\beta_{2}\right) & {\left[2\left(\cos \left(\beta_{2}+\Lambda\right)-\cos \left(\beta_{1}+\Lambda\right)\right)\right.} \\
& -\sin \left(\beta_{1}+\Lambda\right)\left(\tan \beta_{2}+\tan \Lambda+\beta_{1}-\beta_{2}\right) \\
& \left.+\sin \left(\beta_{2}+\Lambda\right)\left(\tan \beta_{2}+\tan \Lambda\right)\right]\end{aligned}$ \\
\hline
\end{tabular}


231 work calculations for the internal stress mechanism. The upper bound estimate of the

232 lateral bearing capacity is therefore determined by equating $\Delta W_{\mathrm{f}}$ and $\Delta W_{\mathrm{p}}$; in

233 dimensionless form (see Table 1), this is expressed as follows:

$$
N_{\mathrm{p}}=\frac{2\left(\Delta W_{\mathrm{p}} / \mathrm{v}_{\mathrm{o}}\right)}{s_{\mathrm{u}}\left(1+\frac{T}{P} \cdot \frac{1}{\lambda^{\prime} R}\right)}
$$
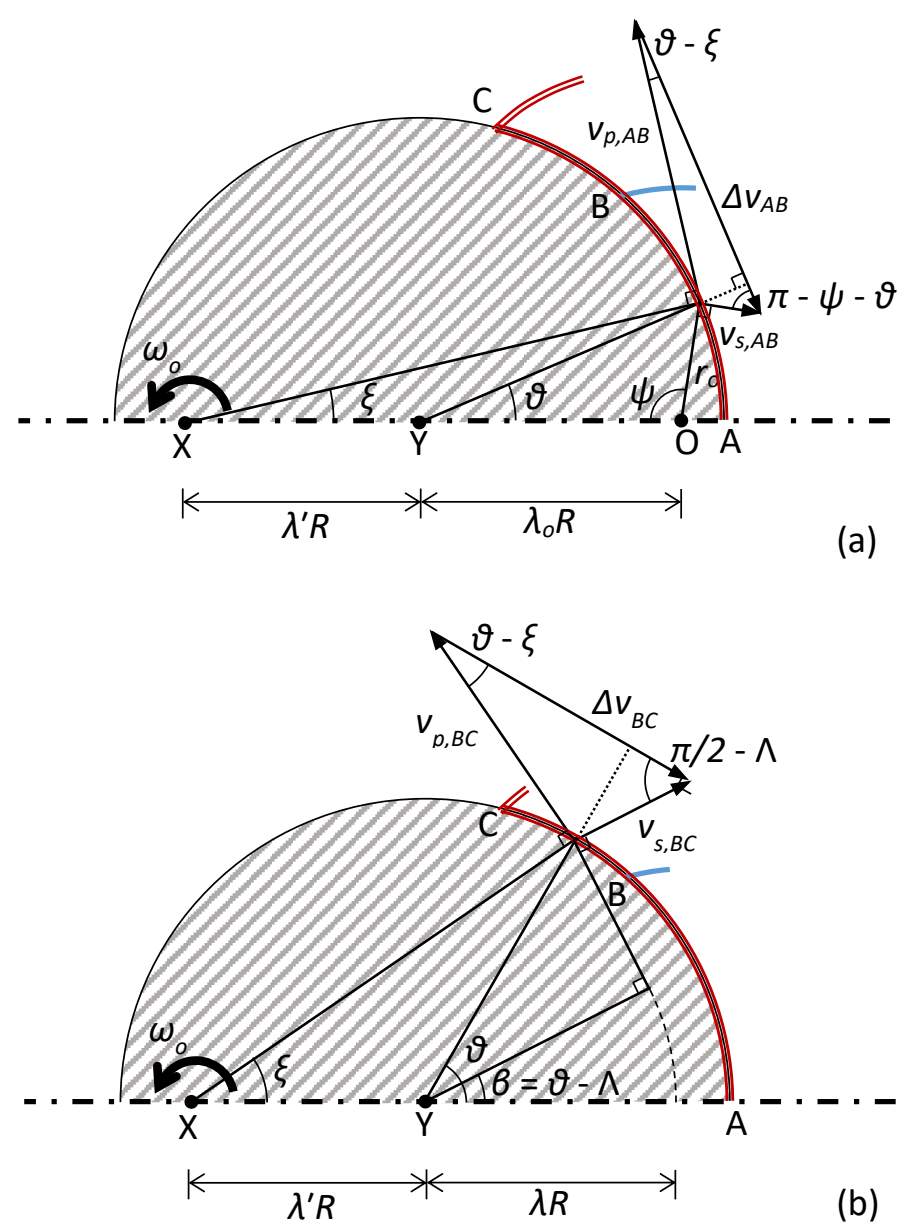

Figure 6: Calculation of velocities (v) and velocity jumps $(\Delta v)$ along discontinuities 
Mechanism I considers no slip at the pile-soil interface on the left-hand side of the pile (CGC' in Figure 5). Slip occurs within the soil along discontinuity CHC', where the full shear strength of the soil $\left(s_{u}\right)$ is mobilised. Consequently, in the limiting case when $\mathrm{CHC}$ ' coincides with CGC' (and point $\mathrm{X}$ coincides with point $\mathrm{Y}$ in Figure 5), corresponding to pure rotation about the pile axis, Mechanism I ignores the actual value of the pile-soil adhesion factor $\alpha$ on the left-hand side of the pile and adopts $\alpha=$ 1 instead. This is only an issue when $n_{T}=1$, in which case a simple purely rotational mechanism (termed 'Mechanism II') involving a single velocity discontinuity along the pile-soil interface provides the minimum upper bound solution for $N_{\mathrm{T}}$, given by Equation (2), which is independent of the applied lateral load. For purely-lateral loading $\left(n_{\mathrm{T}}=0\right)$, Mechanism I slightly over-predicts $N_{\mathrm{p}}$ in comparison to the symmetrical Martin and Randolph (2006) mechanism. The maximum difference is $0.67 \%$, occurring at $\alpha=1$. Although the difference between the solutions of the two mechanisms is very small, the Martin and Randolph (2006) solution (Mechanism III) is adopted for purely-lateral loading henceforth.

In Figure 7, the optimised upper bound mechanisms for $n_{\mathrm{T}}=0.5$ and $\alpha$ ranging between 0.25 and 1 are compared to the incremental shear strains at failure determined using FEA. It can be seen that the optimised upper bound and FEA failure mechanisms show excellent agreement. The associated computed lateral bearing capacity factors are also in excellent agreement with a maximum discrepancy of $0.1 \%$. 
(a)

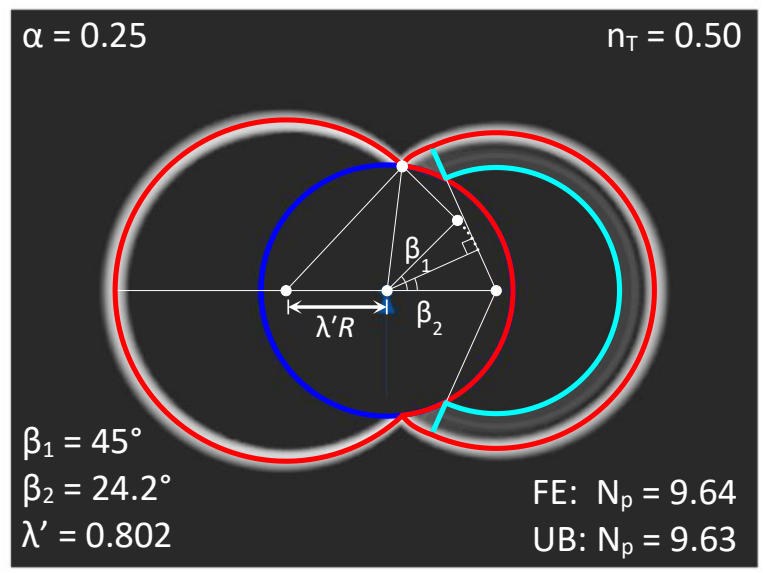

(c)

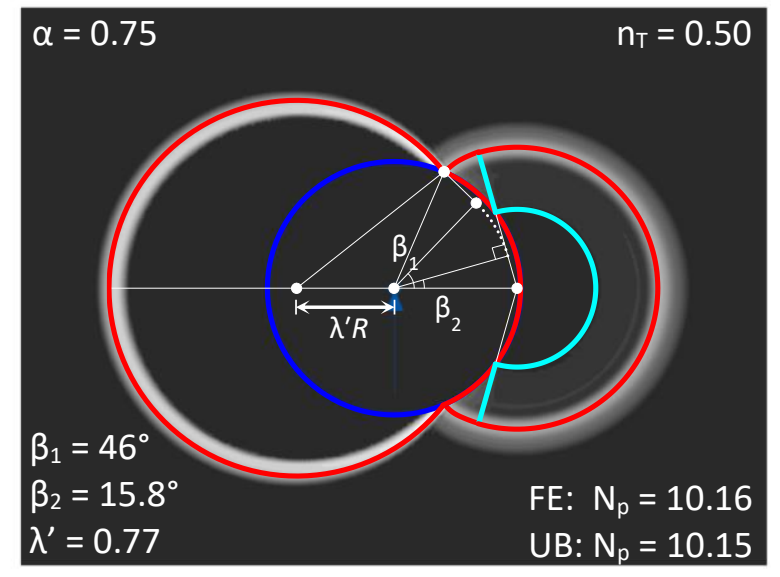

(b)

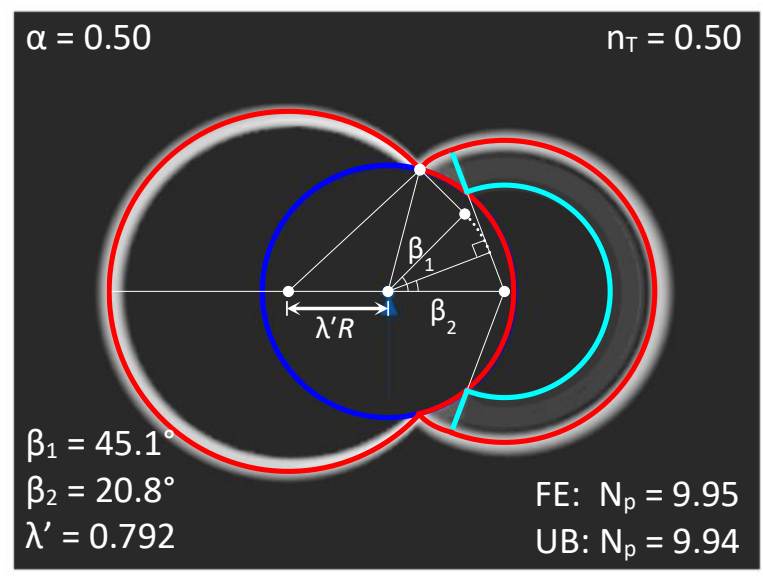

(d)

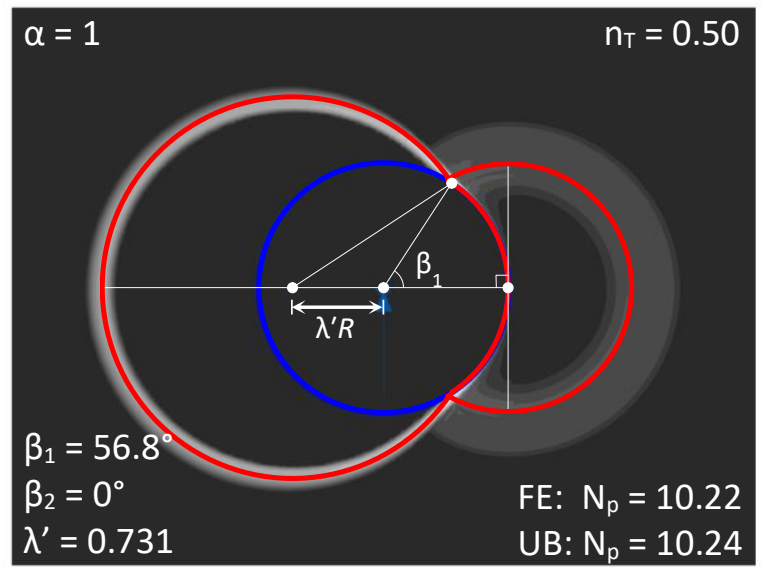

\section{3}

$264 \quad$ Figure 7: Comparison of incremental shear strains determined using FEA and

265 optimum upper bound mechanisms for $\mathrm{n}_{\mathrm{T}}=0.50$ and $\alpha=$ (a) 0.25 , (b) 0.5 , (c) 0.75 and 266

(d) 1 


\section{Lower bound stress field}

For the determination of the lower bounds of the pile failure loads, the stress field shown in Figure 8 is proposed. Similar to the upper bound analysis, a rigid rotating body consisting of the pile and a segment of the soil, is also considered here. The geometry of this rigid region is defined using optimization parameters $\beta_{\mathrm{o}}$ and $\lambda^{\prime}$; the combination of these parameters that maximises the calculated failure load provides the optimum lower bound solution for each analysis. Lower bound estimates of the exact failure loads are determined using a stress field that satisfies the equilibrium equations everywhere in the domain and does not violate the failure criterion (Tresca in this case) anywhere in the domain.

The stress field around the rigid region is geometrically symmetrical about the $\mathrm{x}-\mathrm{x}$ axis and therefore only the upper half of the geometry is presented. It comprises four zones: two zones on the right-hand side of the pile (Zones I and II), which are similar to the stress field used by Randolph and Houlsby (1984), a zone on the left-hand side of the pile (Zone III) and a uniform stress zone (Zone IV) that is separated from the other zones by two stress discontinuities (see Figure 8). Two sets of perpendicular stress characteristics are defined in each zone. A brief description of the stresses in the four stress zones is given below.

Zone I is a stress fan with centre A. The first set of stress characteristics in this zone are straight radial lines, as shown in Figure 8, along which the major principal stress plane angle $\theta_{\mathrm{I}}$ (with respect to the horizontal plane) and the mean stress $p_{\mathrm{I}}$ are equal to:

$$
\theta_{\mathrm{I}}=\theta_{3}+\frac{\pi}{4}
$$




$$
p_{\mathrm{I}}=p_{\mathrm{oR}}+2 s_{\mathrm{u}} \theta_{3}
$$

289 where $p_{\mathrm{OR}}$ is the mean stress at $\theta_{3}=0$ (on the plane of geometrical symmetry). The 290 second set of characteristics are circular arcs with centre at point A.

291

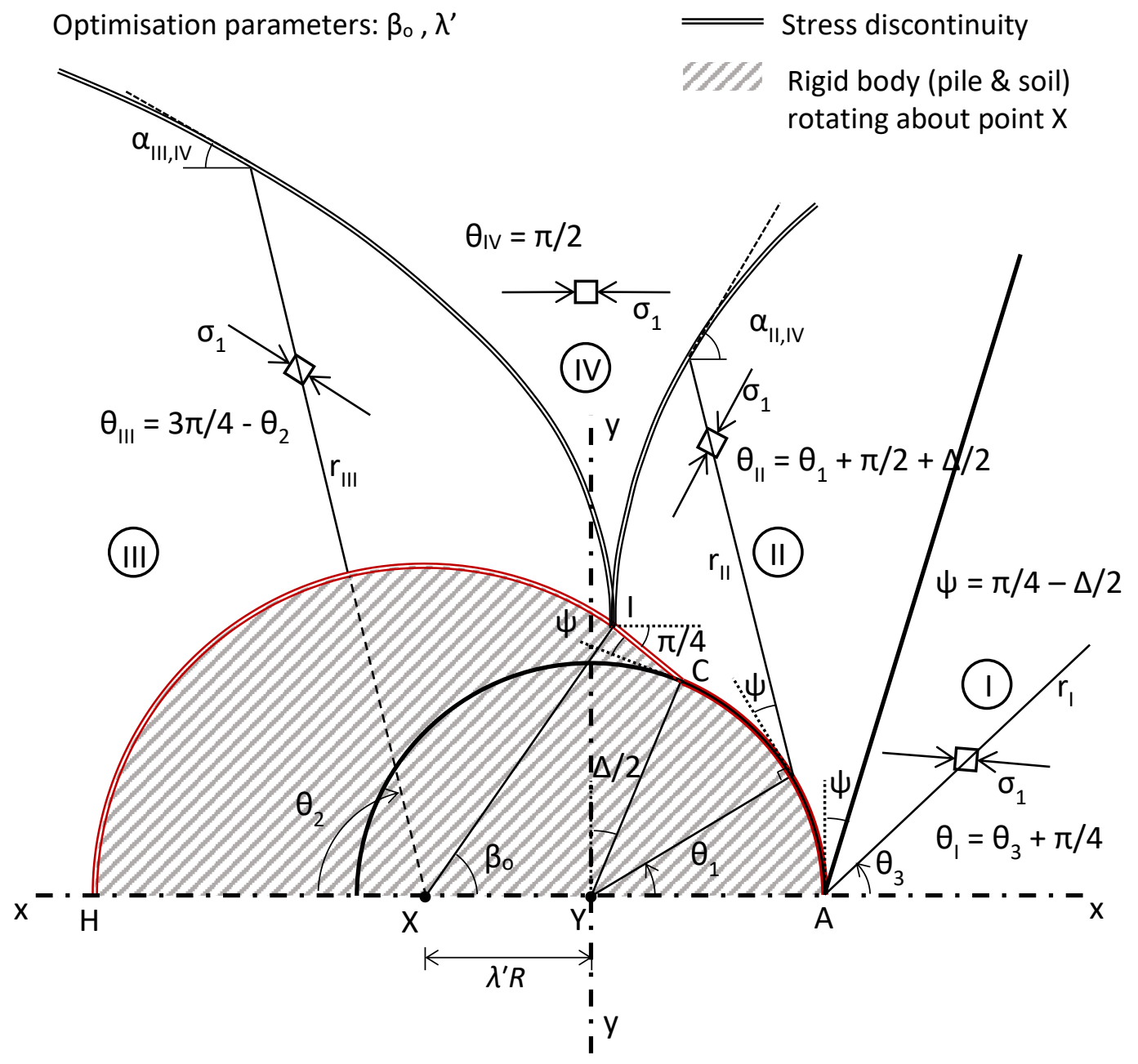

Figure 8: Lower bound stress field for a pile subjected to combined lateral-torsional loading 

stress plane angle $\theta_{\text {II }}$ and the mean stress $p_{\text {II }}$ are equal to:

$$
\begin{aligned}
& \theta_{\mathrm{II}}=\theta_{1}+\frac{\pi}{2}+\frac{\Delta}{2} \\
& p_{\mathrm{II}}=p_{\mathrm{oR}}+2 s_{\mathrm{u}}\left(\theta_{1}+\frac{\pi}{4}+\frac{\Delta}{2}\right)
\end{aligned}
$$

300 In the case of a rough pile $(\alpha=1)$, the stress characteristics are tangential to the pile 301 soil interface $(\psi=0)$.

302 The stress characteristic at the limiting value of $\theta_{\mathrm{II}}=\pi / 2-\Delta / 2$, is inclined at $\pi / 4$ to the 303 horizontal and coincides with the straight section CI of the perimeter of the rigid region 304 with length $L_{\mathrm{CI}}$ defined as follows:

$$
L_{\mathrm{CI}}=R \frac{\lambda^{\prime} \sin \beta+\sin \left(\beta_{\mathrm{o}}-\arccos \lambda-\frac{\pi}{4}\right)}{\sin \left(\frac{\pi}{4}+\beta_{\mathrm{o}}\right)}
$$

305 Zone III is a stress fan with centre at point X. The first set of characteristics are

306 straight lines that extend perpendicularly from the circular perimeter of the rigid

307 region $\mathrm{XIH}$, since the shear strength along this perimeter is equal to $s_{\mathrm{u}}$. The principal 308 stress direction and mean stresses in this region are:

$$
\begin{aligned}
& \theta_{\mathrm{III}}=\frac{3 \pi}{4}-\theta_{2} \\
& p_{\mathrm{III}}=p_{\mathrm{oL}}+2 s_{\mathrm{u}} \theta_{2}
\end{aligned}
$$

309 where $p_{\mathrm{oL}}$ is the mean stress at $\theta_{2}=0$ (on the plane of geometrical symmetry). The 310 second set of characteristics are circular arcs with centre at point $\mathrm{X}$ and radii greater than the radius of the circular part of the rigid region, $L_{\mathrm{XI}}$ : 


$$
L_{\mathrm{XI}}=R \frac{\lambda+\frac{\lambda^{\prime}}{\sqrt{2}}}{\sin \left(\frac{\pi}{4}+\beta_{\mathrm{o}}\right)}
$$

Boundary AC and symmetrical boundary;

$$
\begin{aligned}
P_{\mathrm{AC}}= & \int_{-\left(\frac{\pi}{2}-\frac{\Delta}{2}\right)}^{\frac{\pi}{2}-\frac{\Delta}{2}} \alpha S_{\mathrm{u}} R \cos \theta_{1} \mathrm{~d} \theta_{1} \\
& +\int_{-\left(\frac{\pi}{2}-\frac{\Delta}{2}\right)}^{0}\left[p_{\mathrm{oR}}-2 s_{\mathrm{u}}\left(\frac{\pi}{4}+\frac{\Delta}{2}+\frac{\cos \Delta}{2}-\theta_{1}\right)\right] R \sin \theta_{1} \mathrm{~d} \theta_{1} \\
& +\int_{0}^{\frac{\pi}{2}-\frac{\Delta}{2}}\left[p_{\mathrm{oR}}+2 s_{\mathrm{u}}\left(\frac{\pi}{4}+\frac{\Delta}{2}+\frac{\cos \Delta}{2}+\theta_{1}\right)\right] R \sin \theta_{1} \mathrm{~d} \theta_{1} \\
& =2 s_{\mathrm{u}} R\left[\frac{\pi}{2}-1+\Delta+\left(1-\frac{3 \pi}{2}\right) \sin \frac{\Delta}{2}+2 \cos \frac{\Delta}{2}\left(1+\frac{\cos \Delta}{2}\right)\right]
\end{aligned}
$$

Boundary CI and symmetrical boundary;

$$
\begin{array}{r}
P_{\mathrm{CI}}=2 s_{\mathrm{u}} L_{\mathrm{CI}} \frac{\sqrt{2}}{2}+\left(p_{\mathrm{oR}}+\frac{3 \pi}{2} s_{\mathrm{u}}\right) L_{\mathrm{CI}} \frac{\sqrt{2}}{2}-\left(p_{\mathrm{oR}}-\frac{3 \pi}{2} s_{\mathrm{u}}\right) L_{\mathrm{CI}} \frac{\sqrt{2}}{2} \\
=2 s_{\mathrm{u}} R \frac{\lambda^{\prime} \sin \beta_{\mathrm{o}}+\sin \left(\beta_{\mathrm{o}}-\Lambda-\frac{\pi}{4}\right)}{\sin \left(\beta_{\mathrm{o}}+\frac{\pi}{4}\right)} \frac{\sqrt{2}}{2}\left(1+\frac{3 \pi}{2}\right)
\end{array}
$$


Boundary IH and symmetrical boundary;

$$
\begin{array}{r}
P_{\mathrm{IH}}=\int_{-\left(\pi-\beta_{\mathrm{o}}\right)}^{\pi-\beta_{\mathrm{o}}}-s_{\mathrm{u}} L_{\mathrm{XI}} \cos \theta_{2} \mathrm{~d} \theta_{2}+\int_{-\left(\pi-\beta_{\mathrm{o}}\right)}^{\pi-\beta_{\mathrm{o}}}\left(p_{\mathrm{oL}}+2 s_{\mathrm{u}} \theta_{2}\right) \sin \theta_{2} L_{\mathrm{XI}} \mathrm{d} \theta_{2} \\
=2 s_{\mathrm{u}} R\left(\lambda+\frac{\lambda^{\prime}}{\sqrt{2}}\right) \frac{2 \sin \beta_{\mathrm{o}}+2\left(\pi-\beta_{\mathrm{o}}\right) \cos \beta_{\mathrm{o}}-\sin \beta_{\mathrm{o}}}{\sin \left(\beta_{\mathrm{o}}+\frac{\pi}{4}\right)}
\end{array}
$$

321 The lower bound estimate of $P$ (and therefore $N_{\mathrm{p}}$ ) is determined by summing the

322 forces given by Equations (19), (20) and (21):

$$
N_{\mathrm{p}}=\frac{P_{\mathrm{AC}}+P_{\mathrm{CI}}+P_{\mathrm{IH}}}{s_{\mathrm{u}} D}
$$

323 The limiting torque $T$ at the centre of the pile is determined by summing the

324 components of torque acting on the boundary of the rigid region (see Figure 9):

$$
\begin{aligned}
T=T_{\mathrm{AC}}+T_{\mathrm{IH}} & +T_{\mathrm{CI}}-\lambda^{\prime} R\left(P_{\mathrm{CI}}+P_{\mathrm{IH}}\right) \\
& =\alpha s_{\mathrm{u}} R^{2}(\pi-\Delta) \\
& +2 s_{\mathrm{u}} L_{\mathrm{CI}}\left[R\left(\lambda+\frac{\lambda^{\prime}}{\sqrt{2}}\right)+\frac{3 \pi}{2}\left(\frac{L_{\mathrm{CI}}}{2}-L_{\mathrm{XI}} \sin \left(\beta_{\mathrm{o}}+\frac{\pi}{4}\right)\right)\right] \\
& +2 s_{\mathrm{u}}\left(\pi-\beta_{\mathrm{o}}\right) L_{\mathrm{XI}}^{2}-\lambda^{\prime} R\left(P_{\mathrm{CI}}+P_{\mathrm{IH}}\right)
\end{aligned}
$$




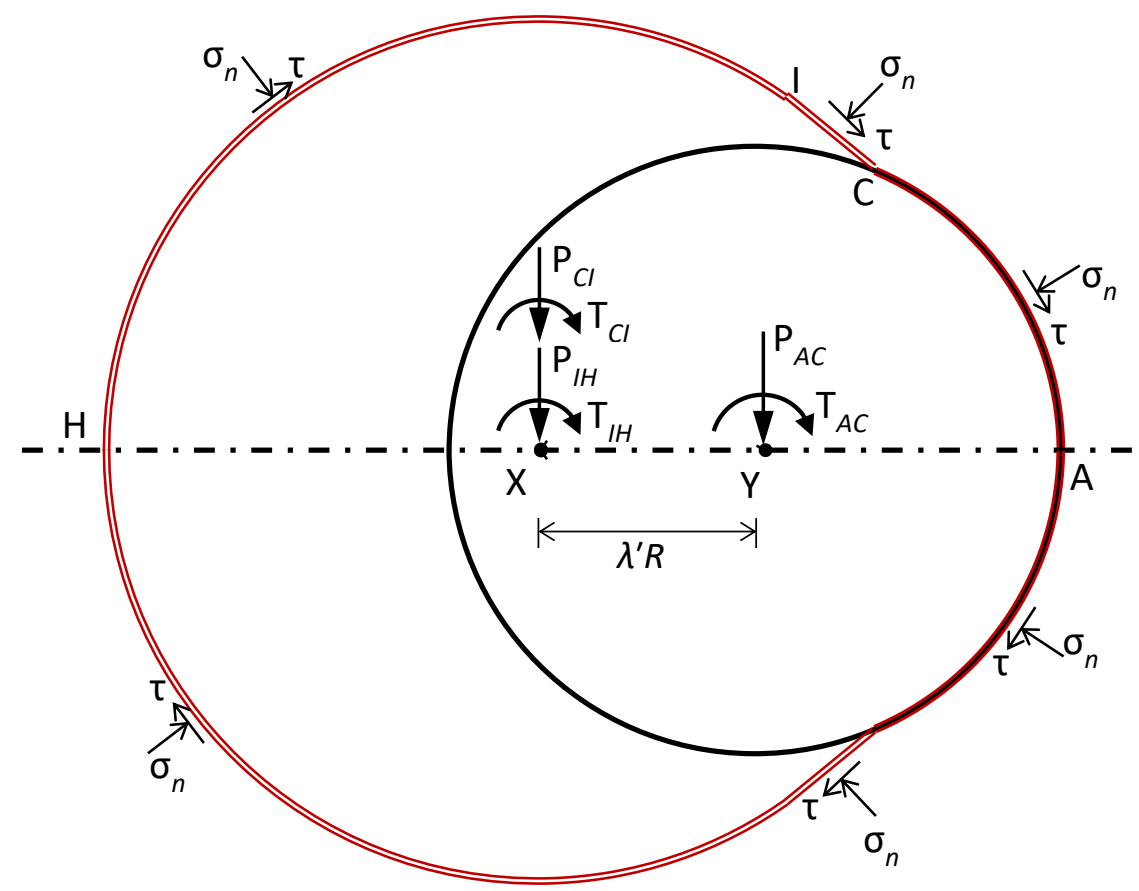

Figure 9: Definition of forces and moments acting on the pile and rigid soil region, and stresses acting on the boundary of the rigid region

For the presented solution to be a true lower bound solution, it must be shown that the stresses do not violate the yield criterion at any point of the domain. This condition is obviously satisfied in Zones I, II and III. The other two regions: the soil part of the rigid body (Figure 8) and Zone IV are discussed below.

The radial stress characteristics of Zone III can be extended into circular region XIH. Although the mean stress along such a radial stress characteristic (dashed line in Figure 8) is constant, as described by Equation (17), the radius of the Mohr's circle of stress can vary continuously from $\mathrm{s}_{\mathrm{u}}$ at the velocity discontinuity IH to zero at point $\mathrm{X}$ such that it does not exceed the pile-soil adhesion $\left(\alpha \cdot s_{u}\right)$ at any point along the pilesoil interface. It follows that the yield criterion is therefore not violated in this region. 
342 Following a procedure similar to that presented by Randolph and Houlsby (1984), it

343 can be shown that the stresses above the two discontinuities illustrated in Figure 8

344 also do not violate the yield criterion and therefore the solution presented above is

345 indeed a lower bound solution. The following equations are derived to determine the 346 inclinations $\alpha_{\mathrm{IV} / \mathrm{I}}, \alpha_{\mathrm{IV} / \mathrm{II}}, \alpha_{\mathrm{IV} / \mathrm{III}}$ and shapes of the two discontinuities:

347 Right-hand stress discontinuity between Zones IV and II $\left(0<\theta_{1} \leq \pi / 2-\Delta / 2\right)$ :

$$
\begin{aligned}
& r_{\mathrm{II}}=\frac{L_{\mathrm{CI}}-R \cos \left(\frac{\pi}{4}-\frac{\Delta}{2}\right)\left[\theta_{1}-\frac{\pi}{2}+\frac{\Delta}{2}+1-\sin \left(\theta_{1}+\frac{\Delta}{2}\right)\right]}{2 \sin ^{2}\left(\frac{\theta_{1}}{2}+\frac{\Delta}{4}\right)} \\
& \alpha_{\mathrm{IV} / \mathrm{II}}=\frac{\theta_{1}}{2}+\frac{\pi}{4}+\frac{\Delta}{4}
\end{aligned}
$$

348 Right-hand stress discontinuity between Zones IV and I $\left(\theta_{1}=0,0 \leq \theta_{3} \leq \pi / 2+\Delta / 2\right)$ :

$$
\begin{aligned}
& r_{\mathrm{II}}=\frac{L_{C I}-R \cos \left(\frac{\pi}{4}-\frac{\Delta}{2}\right)\left[1-\frac{\pi}{2}+\frac{\Delta}{2}-\sin \left(\frac{\Delta}{2}\right)\right]}{2 \sin ^{2}\left(\frac{\pi}{8}-\frac{\theta_{3}}{2}\right)} \\
& \alpha_{\mathrm{IV} / \mathrm{I}}=\frac{\theta_{3}}{2}+\frac{\pi}{8}
\end{aligned}
$$

Left-hand stress discontinuity between Zones IV and III $\left(0 \leq \theta_{2} \leq \pi-\beta_{\mathrm{o}}\right)$ :

$$
\begin{aligned}
& r_{\mathrm{III}}=L_{\mathrm{XI}} \frac{\sin ^{2}\left(\frac{11 \pi}{8}-\frac{\beta_{\mathrm{o}}}{2}\right)}{\sin ^{2}\left(\frac{7 \pi}{8}+\frac{\theta_{2}}{2}\right)} \\
& \alpha_{\mathrm{IV} / \mathrm{III}}=\frac{7 \pi}{8}-\frac{\theta_{2}}{2}
\end{aligned}
$$

350 Based on Equations (24) and (26), the right-hand side discontinuity at point I (Figure 351 8), where both discontinuities originate, is vertical ( $\alpha_{\mathrm{IV} / \mathrm{II}}$ defined in Equation $(24)$ is 
equal to $\pi / 2$ ). The left-hand side discontinuity has an inclination of $3 \pi / 8+\beta_{\mathrm{o}} / 2$. In order to avoid overlapping of the two discontinuities, the inclination (at point I) of the left stress discontinuity must be equal to or less than $\pi / 2$, which is true for any value of $\beta_{\mathrm{o}} \leq \pi / 4$. In fact, it can be shown that when this condition is not satisfied, a "false lower bound" solution is obtained which is greater than the upper bound solution presented in the previous section.

When $\theta_{3} \rightarrow \pi / 4, r_{\text {II }}$ in Equation (25) tends to infinity and similarly when $\theta_{2} \rightarrow \pi / 4, r_{\text {III }}$ in Equation (26) also tends to infinity. Therefore, the two discontinuities tend asymptotically to two lines that pass through the pile centre and are inclined at $+\pi / 4$ for the right-hand side discontinuity and $-\pi / 4$ for the left-hand side discontinuity.

It is straightforward to show using Mohr's circles of stress, that the stress jump $\Delta p$ at any point on a discontinuity depends on the principal stress direction jump $\Delta \theta$ at that point:

$$
\Delta p=-2 s_{\mathrm{u}} \sin (\Delta \theta)
$$

Using Equation (27), the vertical $\sigma_{\mathrm{y}}$ and horizontal $\sigma_{\mathrm{x}}$ stresses can be determined immediately above each discontinuity. Both $\sigma_{\mathrm{y}}$ and $\sigma_{\mathrm{x}}$ decrease monotonically along each discontinuity, from a maximum value at point I to a minimum value where the discontinuities approach their respective asymptotes. The maximum values at point I can be defined as follows:

$$
\begin{aligned}
& \sigma_{\mathrm{x}}=p_{\mathrm{oR}}+\left(\frac{3 \pi}{2}-1\right) s_{\mathrm{u}} \\
& \sigma_{\mathrm{y}}=p_{\mathrm{oR}}+\left(\frac{3 \pi}{2}-3\right) s_{\mathrm{u}}
\end{aligned}
$$




$$
\begin{aligned}
& \sigma_{\mathrm{x}}=p_{\mathrm{oL}}+2\left[\pi-\beta_{\mathrm{o}}+\frac{1}{2}-\sin \left(\frac{3 \pi}{4}-\beta_{\mathrm{o}}\right)\right] s_{\mathrm{u}} \\
& \sigma_{\mathrm{y}}=p_{\mathrm{oL}}+2\left[\pi-\beta_{\mathrm{o}}-\frac{1}{2}-\sin \left(\frac{3 \pi}{4}-\beta_{\mathrm{o}}\right)\right] s_{\mathrm{u}}
\end{aligned}
$$

371 for the left-hand side discontinuity. Since the stresses immediately above the

372 discontinuities must be equal at the common point I, the following relationship

373 between $p_{o R}$ and $p_{o L}$ is obtained by equating Equations (28) and (29):

$$
p_{\mathrm{oR}}-p_{\mathrm{oL}}=2 s_{\mathrm{u}}\left[\frac{\pi}{4}+1-\beta_{\mathrm{o}}-\sin \left(\frac{3 \pi}{4}-\beta_{\mathrm{o}}\right)\right]
$$

374 The minimum values of the stresses are obtained at $\theta_{3}=\pi / 4$ for the right-hand side

375 discontinuity:

$$
\begin{aligned}
& \sigma_{\mathrm{x}}=p_{\mathrm{oR}}+\left(\frac{\pi}{2}+1\right) s_{\mathrm{u}} \\
& \sigma_{\mathrm{y}}=p_{\mathrm{oR}}+\left(\frac{\pi}{2}-1\right) s_{\mathrm{u}}
\end{aligned}
$$

376 Similarly, the minimum values of the stresses are obtained at $\theta_{2}=\pi / 4$ for the left-hand 377 side discontinuity which can be expressed using Equation (30) as follows:

$$
\begin{aligned}
& \sigma_{\mathrm{x}}=p_{\mathrm{oR}}-2 s_{\mathrm{u}}\left[\frac{3}{2}-\beta_{\mathrm{o}}-\sin \left(\frac{3 \pi}{4}-\beta_{\mathrm{o}}\right)\right] \\
& \sigma_{\mathrm{y}}=p_{\mathrm{oR}}-2 s_{\mathrm{u}}\left[\frac{1}{2}-\beta_{\mathrm{o}}-\sin \left(\frac{3 \pi}{4}-\beta_{\mathrm{o}}\right)\right]
\end{aligned}
$$

378 Considering (i) a monotonic reduction of $\sigma_{\mathrm{x}}$ in the $\mathrm{x}$-x direction from the left

379 discontinuity to the right discontinuity, (ii) that the stresses $\sigma_{\mathrm{y}}$ remain constant in the $380 y-y$ direction, and (iii) that as noted above both stresses $\sigma_{\mathrm{x}}$ and $\sigma_{\mathrm{y}}$ decrease along the 381 discontinuities from a maximum at point $\mathrm{I}$, it is evident that the difference $\sigma_{\mathrm{x}}-\sigma_{\mathrm{y}}$ has 382 a maximum of $2 s_{\mathrm{u}}$ immediately above the discontinuities and is less than $2 s_{\mathrm{u}}$ everywhere else. In order to prove that the yield criterion is not violated within region 
IV it needs to be demonstrated that the difference $\sigma_{\mathrm{x}}-\sigma_{\mathrm{y}}$ is also greater than or equal to the minimum value of $-2 s_{\mathrm{u}}$ everywhere in the region. From Equations (28), (31) and (32), it follows that the maximum value of $\sigma_{\mathrm{x}}$ in the region is given by Equation (31) (for $\theta_{3}=\pi / 4$ ) and the maximum value of $\sigma_{\mathrm{y}}$ is given by Equation (28) (point $\mathrm{I}$ in Figure 8 ). The minimum difference is therefore greater than $-2 s_{\mathrm{u}}$. Equations (28) and (31) do not apply simultaneously at any point in the region, and therefore the actual minimum difference is in fact even greater. The yield criterion is therefore not violated anywhere in the domain and the presented solution is a true lower bound solution.

\section{Results and discussion}

Figure 10 shows the dimensionless failure load $\left(N_{\mathrm{p}}\right.$ and $\left.N_{\mathrm{T}}\right)$ interaction diagram for four different values of $\alpha$ determined using FEA as well as the optimum upper and lower bound solutions. The FEA load interaction diagram was determined by 'probing', under load control, at a constant $T / P$ ratio until failure was achieved and by first applying a predefined torque $T$ and then lateral load $P$ until failure. The calculated values of $N_{\mathrm{p}}$ for pertinent values of $\alpha$ and $n_{\mathrm{T}}$ are presented in Table 4. From both Figure 10 and Table 4, it can be seen that the three solutions are in excellent agreement for all cases. Moreover, the upper and lower bound solutions bracket the exact theoretical solutions very closely with a maximum discrepancy of less than $0.5 \%$. For $n_{\mathrm{T}}=0$, the reader is reminded that the optimum upper and lower bound results correspond to the Martin and Randolph (2006) mechanism and the Randolph and Houlsby (1982) lower bound stress field, respectively. It is also worth remarking that since reduced pile - soil adhesion (in the case of $\alpha<1$ ) is only applied to part of 


$$
n_{\mathrm{p}}=0.5+0.5 \sqrt{1-\alpha^{0.7} n_{\mathrm{T}}}
$$
torque. torsional loads as follows:

the pile - soil interface in the upper and lower bound solutions of Figures 5 and 8, respectively, these solutions overestimate pure torsional failure loads. Therefore, a torsion cut-off is applied equal to the theoretical value given by Equation (2), which is in fact both an upper and a lower bound solution.

Figure 10 and Table 4 confirm that the application of torque has a significant influence on the lateral bearing capacity. This influence is greatest for $\alpha=1$ where the maximum reduction in $N_{\mathrm{p}}$ is $50 \%$. By contrast, the maximum reduction in $N_{\mathrm{p}}$ for $\alpha=$ 0.25 is $11 \%$. In addition, as $\alpha$ decreases, the value of $N_{\mathrm{p}}$ for which pile failure transitions from torsional to lateral failure also increases. To further explore the effect of $\alpha$ on the lateral-torsional load interaction, the results in Figure 10 are re-cast in normalised form (i.e. $n_{\mathrm{p}}$ and $n_{\mathrm{T}}$; see Table 1) in Figure 11. It can be seen that the lateral bearing capacity decreases as $n_{\mathrm{T}}$ increases until torsional failure takes place at a limiting $n_{\mathrm{p}}$, equal to 0.5 in the case of $\alpha=1$. As $\alpha$ decreases, the interaction diagram becomes steeper and tends to become vertical as $\alpha$ tends to zero. In the limiting case of $\alpha=0$, clearly torsional failure occurs even for an infinitesimally small applied

Conveniently, the failure load interaction diagram for $\alpha=1$ is similar to a horizontalvertical normalized load interaction diagram for a strip footing founded on undrained soil. Brinch Hansen's (1970) equation for the load inclination factor is modified here to take account of the effect of $\alpha$, and transformed in terms of normalized lateral and 


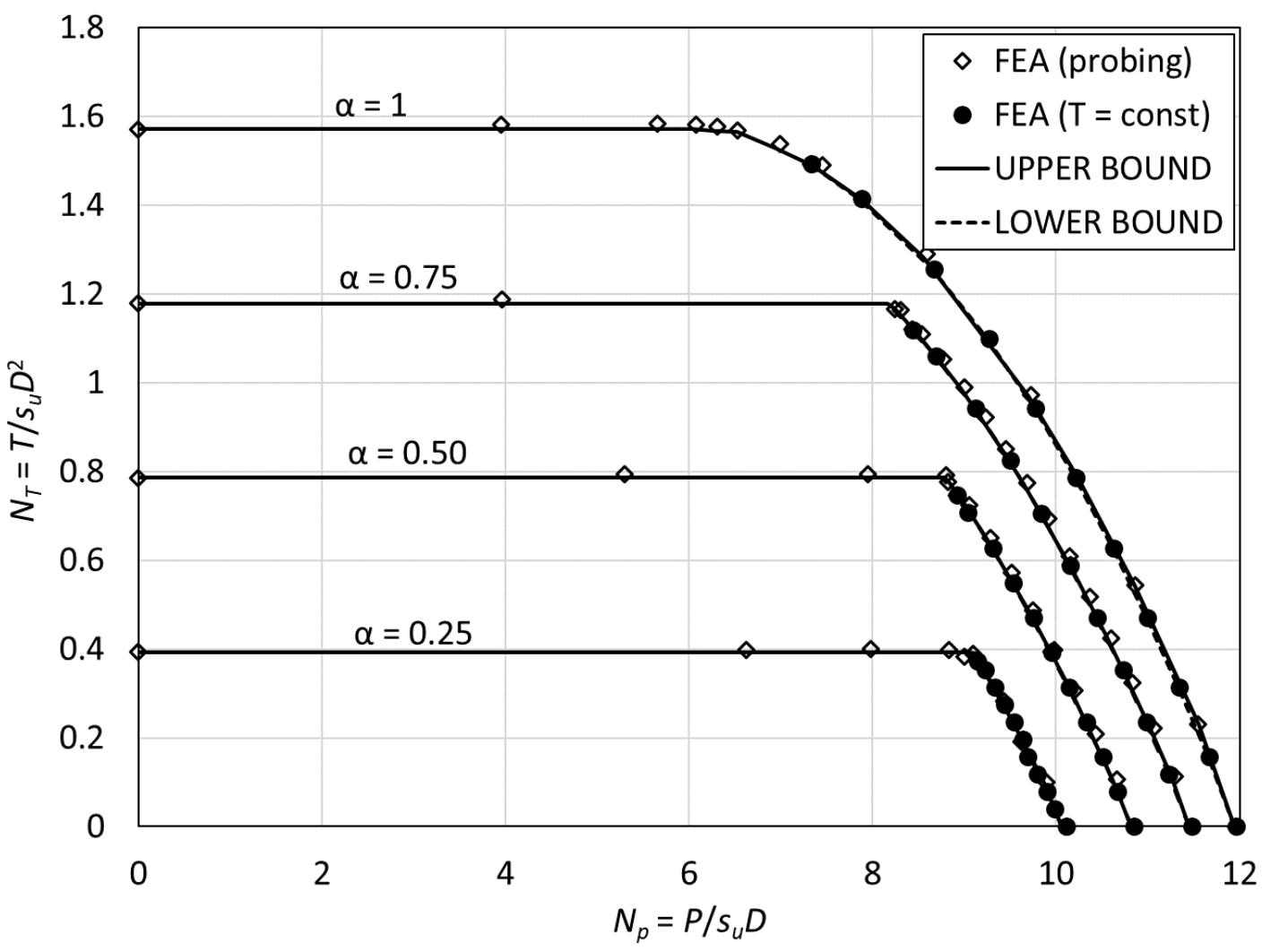

431

Figure 10: Dimensionless failure load interaction diagram

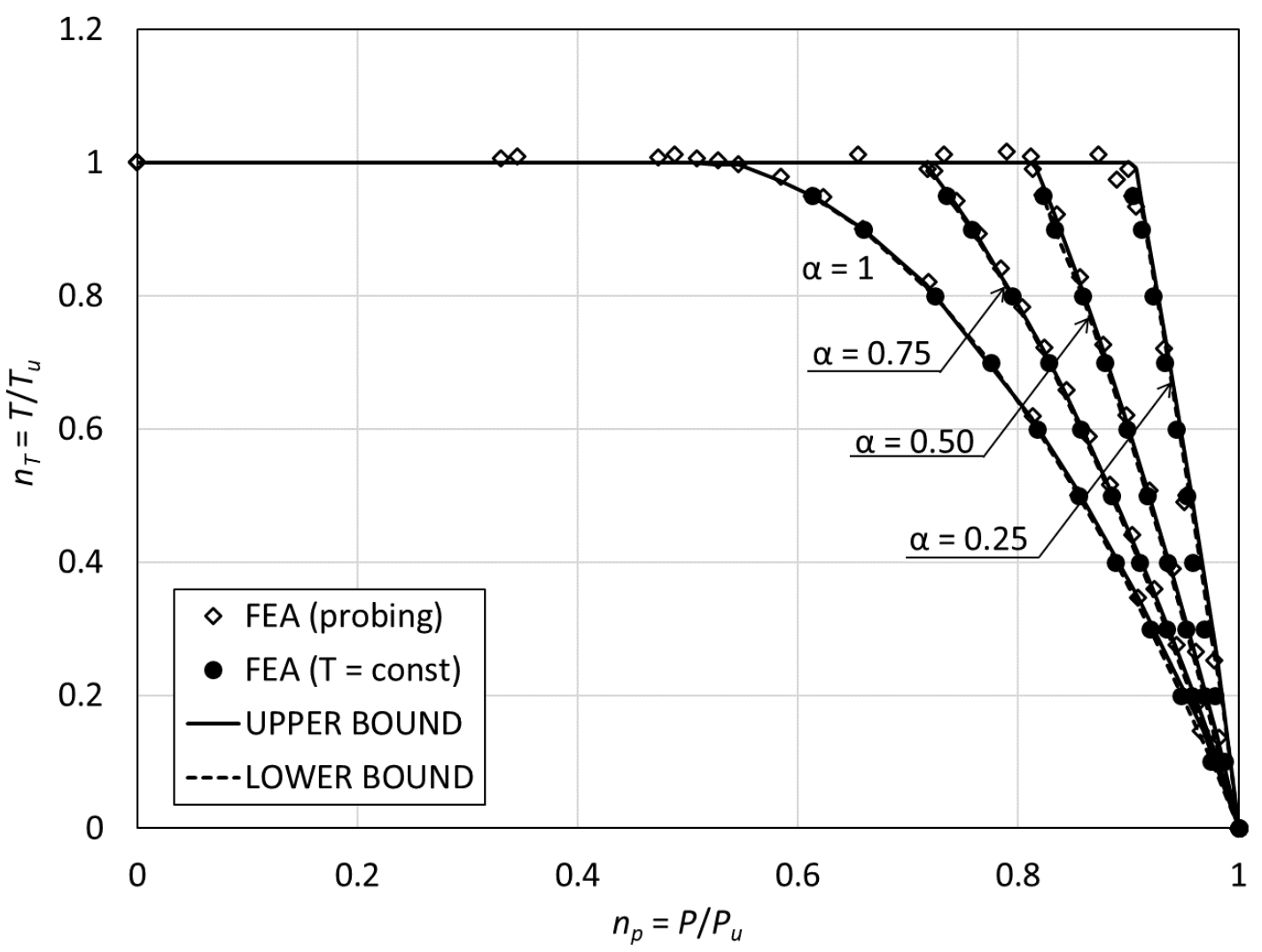

Figure 11: Normalised failure load interaction diagram 


\begin{tabular}{|c|c|c|c|c|c|c|c|c|c|c|c|c|c|c|c|c|}
\hline \multirow[b]{2}{*}{$n_{\mathrm{T}}$} & \multicolumn{4}{|c|}{$\alpha=1$} & \multicolumn{4}{|c|}{$\alpha=0.75$} & \multicolumn{4}{|c|}{$\alpha=0.50$} & \multicolumn{4}{|c|}{$\alpha=0.25$} \\
\hline & FEA & UB & LB & $\begin{array}{c}\text { Eq. } \\
33\end{array}$ & FEA & UB & LB & $\begin{array}{c}\text { Eq. } \\
33\end{array}$ & FEA & UB & LB & $\begin{array}{c}\text { Eq. } \\
33\end{array}$ & FEA & UB & LB & $\begin{array}{c}\text { Eq. } \\
33\end{array}$ \\
\hline 0 & 11.96 & 11.94 & 11.94 & 11.94 & 11.48 & 11.46 & 11.45 & 11.45 & 10.85 & 10.83 & 10.82 & 10.82 & 10.11 & 10.09 & 10.06 & 10.06 \\
\hline 0.1 & 11.66 & 11.71 & 11.65 & 11.63 & 11.23 & 11.24 & 11.23 & 11.21 & 10.67 & 10.68 & 10.67 & 10.65 & 9.98 & 10.01 & 9.98 & 9.96 \\
\hline 0.2 & 11.34 & 11.38 & 11.33 & 11.31 & 10.99 & 10.99 & 10.98 & 10.96 & 10.51 & 10.50 & 10.50 & 10.48 & 9.90 & 9.91 & 9.90 & 9.86 \\
\hline 0.3 & 11.00 & 11.02 & 10.98 & 10.96 & 10.73 & 10.72 & 10.72 & 10.70 & 10.33 & 10.32 & 10.32 & 10.29 & 9.80 & 9.82 & 9.80 & 9.76 \\
\hline 0.4 & 10.62 & 10.65 & 10.61 & 10.59 & 10.45 & 10.44 & 10.44 & 10.42 & 10.15 & 10.14 & 10.13 & 10.11 & 9.69 & 9.73 & 9.71 & 9.66 \\
\hline 0.5 & 10.22 & 10.24 & 10.21 & 10.19 & 10.16 & 10.15 & 10.14 & 10.13 & 9.95 & 9.94 & 9.94 & 9.91 & 9.64 & 9.63 & 9.61 & 9.55 \\
\hline 0.6 & 9.77 & 9.78 & 9.76 & 9.75 & 9.84 & 9.83 & 9.83 & 9.81 & 9.75 & 9.74 & 9.73 & 9.71 & 9.54 & 9.53 & 9.51 & 9.45 \\
\hline 0.7 & 9.27 & 9.27 & 9.25 & 9.24 & 9.50 & 9.49 & 9.49 & 9.47 & 9.54 & 9.53 & 9.52 & 9.49 & 9.44 & 9.43 & 9.41 & 9.34 \\
\hline 0.8 & 8.67 & 8.66 & 8.65 & 8.64 & 9.12 & 9.11 & 9.11 & 9.09 & 9.31 & 9.30 & 9.30 & 9.26 & 9.33 & 9.33 & 9.31 & 9.23 \\
\hline 0.9 & 7.88 & 7.87 & 7.87 & 7.86 & 8.69 & 8.68 & 8.68 & 8.67 & 9.04 & 9.06 & 9.06 & 9.02 & 9.23 & 9.22 & 9.20 & 9.11 \\
\hline 0.95 & 7.33 & 7.31 & 7.31 & 7.30 & 8.44 & 8.45 & 8.45 & 8.43 & 8.92 & 8.94 & 8.93 & 8.90 & 9.14 & 9.17 & 9.15 & 9.05 \\
\hline
\end{tabular}


The lateral bearing capacity factors obtained using Equation (33) are compared to the FEA results as well as the upper and lower bound values in Table 4. It can be seen that this simplified equation provides an excellent approximation of the presented results.

\section{Conclusions}

This paper has described an investigation of the influence of torsion on the ultimate lateral capacity of a single pile wished-in-place in undrained clay. An upper bound failure mechanism and a lower bound stress field were developed that consider simultaneous translation and rotation of a pile located in an ideal rigid - perfectly plastic Tresca soil medium. The soil failure mechanisms obtained from conventional two-dimensional plane strain finite element analyses were used to inform the development of the upper bound approach. The corresponding upper and lower bound solutions were found to be in excellent agreement, bracketing the exact collapse loads with a maximum discrepancy of less than $0.5 \%$. The failure loads obtained from the finite element analyses were also shown to be in excellent agreement with the two plasticity solutions.

Based on the numerical results and the analytical calculations, failure load interaction diagrams were presented. The diagrams revealed that torsion has a significant influence on lateral pile capacity. In the case of full adhesion $(\alpha=1)$, the application of torque was observed to reduce the lateral bearing capacity by up to $50 \%$, before pure rotational failure occurs. This reduction is smaller for smaller values of the adhesion factor. 
459 A simplified empirical equation was also presented for the calculation of lateral

460 bearing capacity as a function of the applied torque. The equation is a modification of

461 an approach for the prediction of the horizontal-vertical load interaction applied to

462 strip footings and was calibrated through curve-fitting the results of the analyses. The

463 empirical approach is in very good agreement with the results of all three employed

464 methods of analysis employed in the paper.

465

\section{References}

467

468

469

470

471

472

473

474

475

476

477

478

479

480

481

Brinkgreve, R.B.J., Kumarswamy, S., Swolfs, W.M. and Floria, F. (2018). Plaxis 2D 2018 user's manual. Plaxis B.V., Netherlands.

Brown, D.A. and Shie, C.F. (1991). Some numerical experiments with a three dimensional finite element model of a laterally loaded pile. Computers and Geotechnics 12, No. 2, 149-162.

Brown, D.A., Morrison, C. and Reese, L.C., (1988). Lateral load behavior of pile group in sand. Journal of Geotechnical Engineering 114, No. 11, 1261-1276.

Georgiadis, K. and Georgiadis, M. (2010). Undrained lateral pile response in sloping ground. J. Geotech. Geoenviron. Engng ASCE 136, No. 11, 1489-1500.

Georgiadis, K., S. W. Sloan, and A. V. Lyamin. (2013a). Ultimate lateral pressure of two side-by-side piles in clay. Géotechnique 63, No. 9, 733-745.

Georgiadis, K., S. W. Sloan, and A. V. Lyamin. (2013b). Undrained limiting lateral soil pressure on a row of piles. Computers and Geotechnics 54, 175-184.

Brinch Hansen, J. (1970). A revised and extended formula for bearing capacity.

Danish Geotechnical Institute Bulletin 28, Danish Geotechnical Institute, 5-11. 
Byrne, B.W. and Houlsby, G.T. (2003). Foundations for offshore wind turbines. Philosophical Transactions of the Royal Society of London. Series A: Mathematical, Physical and Engineering Sciences, 361, No. 1813, 2909-2930.

Herrera, R. (2001). Determine optimum depths of drilled shafts subjected to combined torsion and lateral loads using the centrifuge. Master's thesis, University of Florida, Gainesville, Florida.

$\mathrm{Hu}, \mathrm{Z}$. (2003). Determining the optimum depth of drilled shafts subject to combined torsion and lateral loads in saturated sand from centrifuge testing (Doctoral dissertation, University of Florida).

Hu, Z., McVay, M., Bloomquist, D., Herrera, R., and Lai, P. (2006). Influence of torque on lateral capacity of drilled shafts in sands. Journal of Geotechnical and Geoenvironmental engineering 132, No. 4, 454-464.

Jeanjean P. (2009). Re-Assessment of P-Y curves for soft clays from centrifuge testing and finite element modeling. Proceedings of the Offshore Technology Conference, Houston, TX, USA, paper OTC 20158, 1-23.

Martin, C.M. \& Randolph, M.F. (2006). Upper bound analysis of lateral pile capacity in cohesive soil. Géotechnique 56, No. 2, 141-145.

Matlock, H. (1970). Correlations for design of laterally loaded piles in soft clay. Proceedings of the 2nd offshore technology conference, Houston, TX, USA, paper OTC 1204, 577-594.

Murff, J.D. \& Hamilton, J.M., (1993). P-ultimate for undrained analysis of laterally loaded piles. Journal of Geotechnical Engineering, 119, No. 1, 91-107. 
504

505

506

507 508

509

510

511

512

513

514

515

516

517

518

519

520

521

522

523

524

525

Nimityongskul, N., Kawamata, Y., Rayamajhi, D., \& Ashford, S. A. (2017). Fullscale tests on effects of slope on lateral capacity of piles installed in cohesive soils. Journal of Geotechnical and Geoenvironmental Engineering, 144, No. 1, 04017103.

Randolph, M.F. and Houlsby, G.T. (1984). The limiting pressure on a circular pile loaded laterally in cohesive soil. Geotechnique 34, No. 4, 613-623.

Randolph, Mark F. (2003) Science and empiricism in pile foundation design. Géotechnique 53, No. 10, 847-875.

Reddy, S.C. and Stuedlein, A.W. (2017). Serviceability limit state reliability-based design of augered cast-in-place piles in granular soils. Canadian Geotechnical Journal 54, No. 12, 1704-1715.

Sheil, B., McCabe, B., Comodromos, E.M. and Lehane, B.M. (2018). Pile groups under axial loading: an appraisal of simplified non-linear prediction models. Géotechnique 69, No. 7, DOI: 10.1680/jgeot.17.r.040.

Thiyyakkandi, S., McVay, M., Lai, P. and Herrera, R. (2016). Full-scale coupled torsion and lateral response of mast arm drilled shaft foundations. Canadian Geotechnical Journal 53, No. 12, 1928-1938.

Thiyyakkandi, S., McVay, M., Lai, P. and Herrera, R. (2017). Suitability of jetted and grouted precast pile for supporting mast arm structures. Canadian Geotechnical Journal 54, No. 9, 1231-1244

Yang, Z. and Jeremic, B. (2002). Numerical analysis of pile behaviour under lateral loads in layered elastic-plastic soils. Int. J. Numer. Anal. Methods Geomech. 26, 1385-1406. 
526 Yu, J., Huang, M., \& Zhang, C. (2015). Three-dimensional upper-bound analysis for

527 ultimate bearing capacity of laterally loaded rigid pile in undrained clay. Canadian

528 Geotechnical Journal, 52, No. 11, 1775-1790.

529 Zhang, C., White, D., \& Randolph, M. (2010). Centrifuge modeling of the cyclic

530 lateral response of a rigid pile in soft clay. Journal of Geotechnical and

531 Geoenvironmental Engineering, 137, No. 7, 717-729.

532 Zhang, Y., \& Andersen, K. H. (2019). Soil reaction curves for monopiles in clay.

533 Marine Structures, 65, 94-113.

534

535

536

537

538

539 NOTATION

$540 \quad D$ : pile diameter

$541 E A$ : axial stiffness

$542 \quad E l$ : bending stiffness

$543 \quad E_{u}:$ undrained modulus of elasticity

$544 \quad n_{p}:$ normalized lateral failure load per unit length

$545 N_{p}$ : lateral bearing capacity factor

$546 \quad N_{p u}$ : ultimate lateral bearing capacity factor

$547 \quad n_{T}:$ normalized torque per unit length

$548 \quad N_{T}$ : torsional bearing capacity factor 
$550 \quad P$ : lateral failure load per unit length

$551 \quad P_{u}: \quad$ ultimate lateral failure load per unit length

$552 R:$ pile radius

$553 s_{u}: \quad$ undrained shear strength

$554 \quad T: \quad$ torque per unit length

$555 T_{u}: \quad$ ultimate torque per unit length

$556 \quad v_{o}:$ pile lateral velocity

$557 v, \Delta v$ : internal velocities of failure mechanism

$558 \alpha:$ adhesion factor

$559 \beta_{1}, \beta_{2}, \lambda^{\prime}, \beta_{0}$ : geometrical optimization parameters

$560 \Delta W_{p}$ : work done by internal stresses

$561 \Delta W_{f}$ : work done by external forces

$562 \quad v_{u}:$ undrained Poisson's ratio

$563 \tau_{\mathrm{i}}:$ pile-soil interface adhesion

$564 \omega_{0}$ : pile lateral velocity

565

\section{Figure caption list}

567 Figure 1: Schematic plan view of a pile section embedded in undrained clay -

568 Definition of failure loads and associated translational and rotational velocities.

569 Figure 2: (a) Finite element mesh adopted for this study showing increased mesh

570 density in zone immediately surrounding pile; 12876 elements. (b) Detail of mesh

571 around the pile.

572 Figure 3: Contours of incremental soil displacements at failure for a laterally loaded

573 pile with different levels of applied torsion; $\alpha=0.25,0.5,0.75$ and 1 . 
574 Figure 4: Contours of incremental shear strains at failure for a laterally loaded pile 575 with different levels of applied torsion; $\alpha=0.25,0.5,0.75$ and 1.

576 Figure 5: Upper bound failure mechanism for a pile subjected to combined lateral577 torsional loading

578 Figure 6: Calculation of velocities (v) and velocity jumps $(\Delta \mathrm{v})$ along discontinuities 579 (a) $\mathrm{AB}$ and (b) BC

580 Figure 7: Comparison of incremental shear strains determined using FEA and

581 optimum upper bound mechanisms for $\mathrm{n}_{\mathrm{T}}=0.50$ and $\alpha=$ (a) 0.25 , (b) 0.5 , (c) 0.75 and 582 (d) 1

583 Figure 8: Lower bound stress field for a pile subjected to combined lateral-torsional 584 loading

585 Figure 9: Definition of forces and moments acting on the pile and rigid soil region, 586 and stresses acting on the boundary of the rigid region

587 Figure 10: Dimensionless failure load interaction diagram

588 Figure 11: Normalised failure load interaction diagram

\section{Table caption list}

592 Table 1: Parameter normalisation adopted in this study

593 Table 2: Ultimate bearing capacity factors for different values of $\alpha$ for purely-lateral 594 or purely-torsional loading.

595 Table 3: Calculation of work done by internal stresses

596 Table 4: Lateral bearing capacity factors $N_{p}$ for different normalised torsional loads 597 and adhesion factors 